\title{
A wavelength-dispersive instrument for characterizing fluorescence and scattering spectra of individual aerosol particles on a substrate
}

Donald R. Huffman et al.

Correspondence to: J. Alex Huffman (alex.huffman@du.edu)

The copyright of individual parts of the supplement might differ from the CC-BY 3.0 licence. 


\begin{tabular}{|c|c|c|c|c|c|c|c|c|}
\hline \multicolumn{3}{|c|}{ Camera } & & \multirow[b]{2}{*}{$\begin{array}{c}\text { Detector } \\
\text { type }\end{array}$} & \multirow[b]{2}{*}{$\begin{array}{c}\text { Number of } \\
\text { pixels } \\
\left(x 10^{6}, \mathrm{Mp}\right)\end{array}$} & \multirow[b]{2}{*}{$\begin{array}{l}\text { Pixel matrix } \\
(\mathrm{L} x \mathrm{H})\end{array}$} & \multirow[b]{2}{*}{$\begin{array}{c}\text { Pixel size } \\
(\mathrm{L} x \mathrm{H}) \\
\mu \mathrm{m}\end{array}$} & \multirow[b]{2}{*}{ Citation } \\
\hline Manufacturer & Model & Туре & $\begin{array}{c}\text { Color / } \\
\text { Monochrome }\end{array}$ & & & & & \\
\hline Canon & Powershot A2300 HD & Point-and-Shoot & Color & CCD & 15.9 & $4608 \times 3456$ & $1.3 \times 1.3$ & [1] \\
\hline Lumenera & Infinity $2-1 R$ & Research microscopy & Monochrome & CCD & 1.45 & $1392 \times 1040$ & $4.6 \times 4.6$ & [2] \\
\hline Apple & iPhone 5s & Smartphone & Color & CMOS & 8.0 & 3264 x 2448 & $1.5 \times 1.5$ & [3] \\
\hline
\end{tabular}

Table S1: Summary of information regarding cameras discussed in the manuscript text.

Table citations:

[1] http://imaging-resource.com/PRODS/canon-a2300/canon-a2300DAT.HTM

[2] http://www.lumenera.com/resources/documents/datasheets/microscopy/infinity-2-1datasheet.pdf

[3] http://www.gsmarena.com/apple_iphone_5s-5685.php 

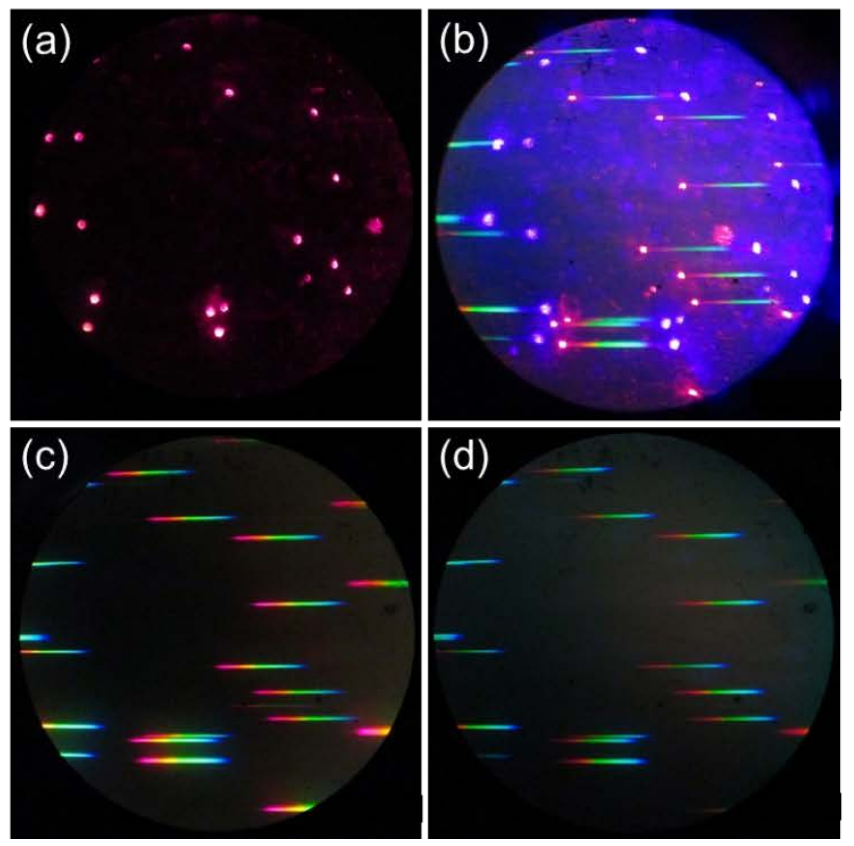

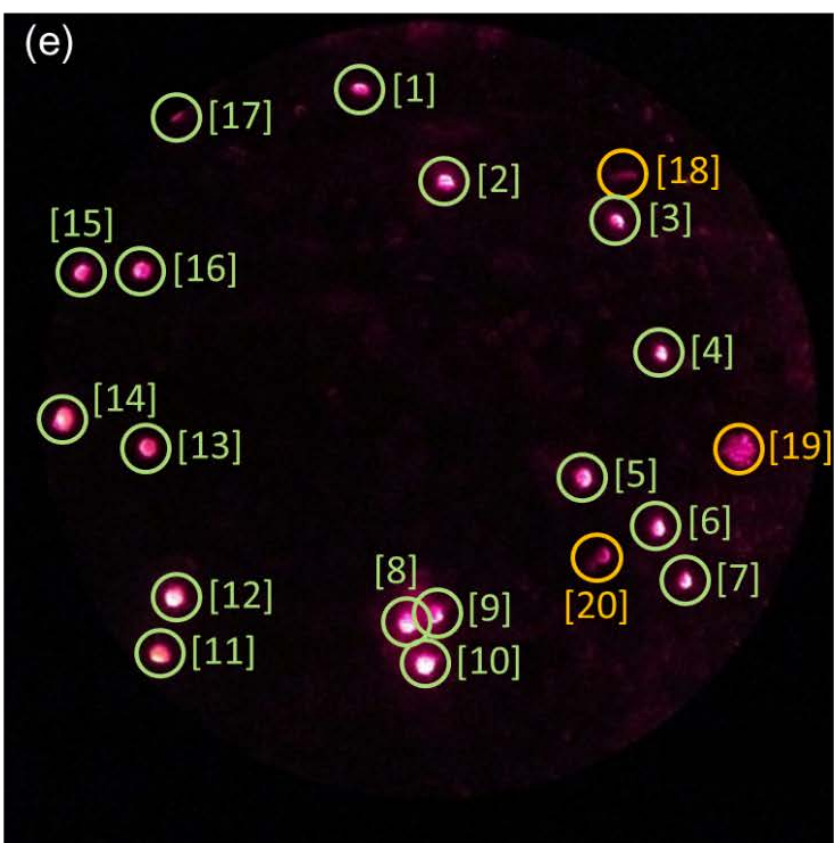

Figure S1: Duplicate of Figure 2 from the manuscript, with panel (a) blown up and shown to the right as panel (e) with each particle circled and numbered for unambiguous discussion (below). Panels (a-d): Four-panel progression of images acquired for a given scene of paper mulberry pollen particles collected onto a glass microscope slide, analyzed using 10x objective. Scale is the same in each figure, with each horizontal swath of color approximately $10 \mu \mathrm{m}$ in height. (a) Dark field image of particles illuminated by monochromatic red laser light $(\theta=0)$. (b) Particles illuminated with both violet $(405 \mathrm{~nm})$ and red $(650 \mathrm{~nm})$ diode lasers. Fluorescence spectra of individual particles showing image taken without use of blocking filter. (c) White light illumination with tungsten filament bulb. (d) Fluorescent emission with excitation from violet diode laser, but using blocking filter to remove violet laser point. Canon Powershot A2300 HD camera utilized offers 4608 x 3456 square pixels $1.3 \mu \mathrm{m}$ in size.

Supplemental description of figure: Careful counting of particles in the four images in Figure 2 (or Fig. S1) may give the reader a better idea of the different information content of each panel as well as showing that almost all of the particle are of similar nature. Twenty (20) particles are highlighted in panel (e), and seventeen (17) show corresponding fluorescence spectra in panel (d). These are numbered 1-17 and highlighted by light green circles. Particles 15 and 16 occur at nearly the same vertical position, and so their fluorescence swaths are superimposed and difficult to separate. Three additional particles (18-20) do not have matching fluorescence spectra and are highlighted in orange. Particle 19 is larger and unusually fuzzy, suggesting it is not the same type of particle as the paper mulberry pollen grains shown. Particles 18 and 20 are both relatively dim even in panel (a), suggesting that they may be small or thin particles, or may be smudges or optical artifacts. They could also be examples of small particles exhibiting fluorescence emission lower than the limits of detectability for fluorescence and scattering discussed, as later in this paper. 

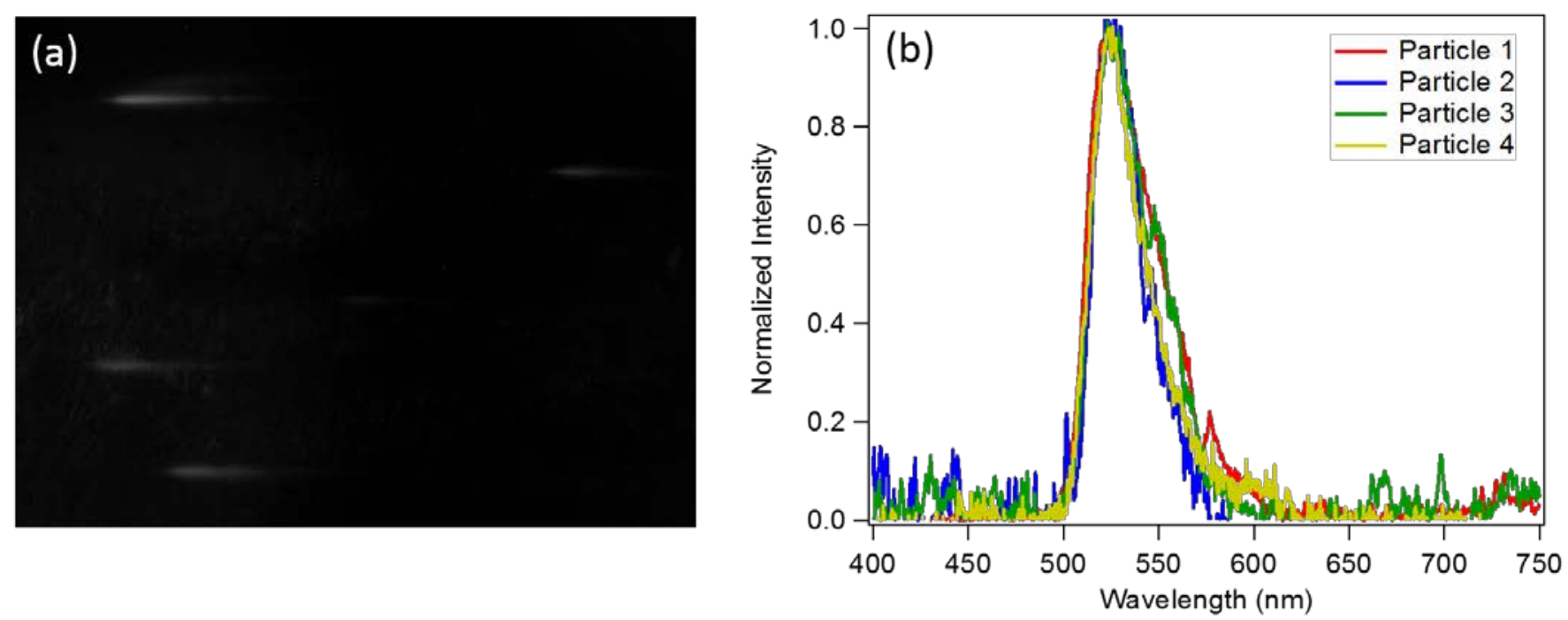

Figure S2: Micrograph image (40x objective) and associated spectra from $0.96 \mu \mathrm{m}$ polystyrene latex spheres doped with dragon green fluorophore (Bangs Laboratories, Inc.). Emission wavelength calibrated using $450 \mathrm{~nm}$ and $650 \mathrm{~nm}$ laser points. All spectra were normalized to 1.0 maximum peak height. 